\title{
Neutral theory: applicability and neutrality of using generic health-related quality of life tools in diseases or conditions where specific tools are available
}

Ravi Jandhyala ${ }^{1,2}$ (D)

\begin{abstract}
Background: Health-related quality of life (HRQoL) tools are limited by the indicators included in the construct and variation in interpretation by different researchers. Neutral Theory describes the ideal construct that includes all relevant indicators and, therefore, complete accuracy, or neutrality. Neutral Theory can thereby provide the framework to develop or test constructs. To assess the application of Neutral Theory, the neutrality of generic tools (SF-36 and EQ-5D) at measuring HRQoL was compared to disease/condition-specific tools, with the latter considered surrogates for the Neutral construct.
\end{abstract}

Methods: Full descriptions of all disease/condition-specific HRQoL tools published on PubMed (to 01-Jul-19) were sourced. For each tool, the number of items with and without a direct match within the SF-36 and EQ-5D was recorded and the sensitivity/specificity calculated.

Results: The SF-36 and EQ-5D did not achieve a sensitivity/specificity both $>50 \%$ against any of the 163 disease/ condition-specific tools identified. At 20\% prevalence of poor HRQoL, the false positive rate (FPR) was $>75 \%$ for all but two tools against the SF-36 and six tools against the EQ-5D. Increasing poor HRQoL to 80\%, 47 tools for the SF36 and 48 tools for the EQ-5D had a FPR $<50 \%$. For rare disease tools $(<1 / 2000$ population; $n=17)$, sensitivity/ specificity ranged from 0 to 40\%/5-31\% for the SF-36 and 0-22\%/29-100\% for the EQ-5D. For non-rare $(n=75)$ and symptom-specific tools $(n=71$ ) sensitivity/specificity was: 0-100\%/0-100\% (SF-36) and 0-50\%/0-100\% (EQ-5D); and 0-60\%/0-19\% (SF-36) and 0-25\%/0-100\% (EQ-5D), respectively. No concordance was recorded for 18\% (2/11) of results from studies of rare disease tools versus the SF-36 (no data vs EQ-5D). For non-rare, disease-specific tools, results were discordant for 30\% (25/84) and 35\% (23/65) of studies against the SF-36 and EQ-5D, respectively. For symptom-specific tools, corresponding results were $36 \%$ (24/66) and 16\% (5/31).

Conclusions: Generic HRQoL tools appear poorly correlated with disease/condition-specific tools, which indicates that adoption of Neutral Theory in the development and assessment of HRQoL tools could improve their relevance, accuracy, and utility in economic evaluations of health interventions.

Keywords: SF-36, EQ-5D, Utility, Patient-reported outcomes (PRO), Health-related quality of life, Construct, Neutral theory

\footnotetext{
Correspondence: ravi@medialis.co.uk

${ }^{1}$ Medialis Limited, Banbury, UK

${ }^{2}$ Centre for Pharmaceutical Medicine Research, Institute of Pharmaceutical

Science, Faculty of Life Science \& Medicine, King's College London, London,

UK
}

C C The Author(s). 2021 Open Access This article is licensed under a Creative Commons Attribution 4.0 International License, which permits use, sharing, adaptation, distribution and reproduction in any medium or format, as long as you give appropriate credit to the original author(s) and the source, provide a link to the Creative Commons licence, and indicate if changes were made. The images or other third party material in this article are included in the article's Creative Commons licence, unless indicated otherwise in a credit line to the material. If material is not included in the article's Creative Commons licence and your intended use is not permitted by statutory regulation or exceeds the permitted use, you will need to obtain permission directly from the copyright holder. To view a copy of this licence, visit http://creativecommons.org/licenses/by/4.0/ The Creative Commons Public Domain Dedication waiver (http://creativecommons.org/publicdomain/zero/1.0/) applies to the data made available in this article, unless otherwise stated in a credit line to the data. 


\section{Background}

Observation of a construct, such as a quality of life tool, first requires conceptualisation of the construct at a theoretical level followed by its operationalisation at an empirical level. Operationalisation involves selecting indicators to be measured in the observation of the construct. Both are vulnerable to variation in interpretation by different researchers and can result in a divergence in their measurement of the 'same' construct. A new theory recently proposed is that of a 'Neutral Observer', which provides a framework on which a determination of the neutrality, or accuracy, of an observation of a given construct can be based [1]. Neutral Theory represents the ideal and assumes a Neutral or exhaustive list of relevant indicators in the construct, whereby the sensitivity and specificity are both 1 (i.e., 100\% accurate). The operationalisation of constructs using disease-specific indicators can perhaps be considered closer to achieving neutrality than those based on generic observations.

Understanding the impact of treatment on patients' quality of life is a pivotal component in the economic evaluation of health interventions. There is, however, no universally agreed definition of the construct of quality of life, with the one provided by the World Health Organization (WHO) perhaps the most commonly cited: "an individual's perception of their position in life in the context of the culture and value systems in which they live and in relation to their goals, expectations, standards and concerns" [2]. This broad definition includes the person's physical health, psychological state, personal beliefs, social relationships and their relationship to salient features of their environment. The WHO definition, and other similar ones, were influential in the concept of health-related quality of life (HRQoL), which refers to how well a person functions in their life and his or her perceived well-being in physical, mental, and social domains of health [3].

Two independently operationalised tools that are frequently used to objectively assess HRQoL are: the Medical Outcomes Study Short Form family of measures (e.g. SF-36 [4, 5]) and the EuroQol five-dimensional (EQ-5D) [6, 7]. Both of these tools capture HRQoL (or, strictly speaking, health status for the EQ-5D $[6,8]$ ) across a series of domains or dimensions: vitality, physical functioning, bodily pain, general health perceptions, physical functioning, emotional functioning, social functioning, and mental health in the SF-36 [5]; and mobility, self-care, usual activities, pain/discomfort, and anxiety/ depression in the EQ-5D [7].

Generic HRQoL tools have been widely adopted in Health Technology Assessments (HTAs), with the National Institute for Health and Care Excellence (NICE) in the UK recommending use of the EQ-5D in its Technology Appraisals [9]. Generic HRQoL tools, by their nature, depict aspects of well-being and quality of life from the patients' point of view across all diseases and, therefore, have utility in population-level studies as well as informing comparisons between diseases. While this allows for potentially more consistent, transparent and predictable decision-making, it is open to criticism, as generic measures may be insensitive or fail to capture important aspects of health for a specific disease or condition [10]. Disease- or condition-specific HRQoL tools have the advantage of being clinically relevant to the health problem and responsive to clinically important changes in state, such as the impact of treatment. Conversely, this specificity complicates comparisons with the general population and across treatments for different diseases, limiting their application in HTAs.

This study aimed to apply Neutral theory in assessing the neutrality, or accuracy, and applicability of generic tools (SF-36 and EQ-5D) at measuring HRQoL in diseases or conditions where there is a specific tool available, to act a surrogate for the Neutral list in the measurement of HRQoL.

\section{Methods \\ Identification of disease- or condition-specific health- related quality of life tools}

A literature search was performed to identify all published disease- and condition-specific HRQoL tools. Medline (PubMed) was searched through 01 July 2019 using the following terms: ["patient reported outcome" OR "PRO" OR "Quality of life" OR "QoL" AND "disease specific" OR "condition specific"]; limit: [English language]. Two reviewers undertook the search, with initial screening of abstracts and titles conducted using the semi-automated Rayyan tool (https://rayyan.qcri.org/) [11]. Full descriptions of the identified disease/condition-specific HRQoL tools were sourced as were the SF36 and EQ-5D. In addition, all original studies where HRQoL was assessed using a disease/condition-specific HRQoL tool and the SF-36 and/or the EQ-5D were reviewed.

\section{Inclusion of appropriate domains and items}

The risk that the generic tools (SF-36 and EQ-5D) might include irrelevant domains or items or exclude relevant domains or items for a specific disease or condition was assessed. Firstly, for each condition- or disease-specific tool the number of items with and without a direct match to the SF-36 and EQ-5D was recorded (for the EQ-5D, it was permitted for each of the five questions to cover more than one item in each disease/condition-specific tool). The sensitivity and specificity of the generic tool versus the disease/condition-specific tool was then calculated as follows. True positives represented items captured in both the disease/condition specific and 
generic tool; false positives, those captured in the generic tool, but not in the disease/condition specific tool; and false negatives, those captured in the disease/condition specific tool, but not in the generic tool. Since it is not possible to know if the disease/condition specific tool fully captures all relevant items or domains, the true negative fraction was assumed to be 0.9 (i.e. an arbitrary $10 \%$ missing). Sensitivity/specificity results were further stratified into rare diseases (defined as affecting $<1$ in 2000 population) [12], non-rare diseases $(\geq 1$ in 2000 population), and symptom-specific tools (i.e. those that cover symptoms [e.g. urological symptoms; respiratory problems $e t c$ ] that might be present in multiple diseases/ conditions).

The potential for misclassification of patients' HRQoL by a generic tool was expressed as the median proportion of false positives and false negatives (with 95\% prediction intervals), based on 1000 studies, with prevalence of poor HRQoL set at 20,50, and $80 \%$.

\section{Concordance of quality of life scores}

For each of the studies comparing a disease/conditionspecific tool with the SF-36 and/or EQ-5D, a measure of concordance of the results was assigned. No (none) concordance was assigned if a significant impact on HRQoL was seen with the disease/condition specific tool, but no change or the opposite impact was seen with the generic tool (or vice versa); Moderate concordance if HRQoL impact was scored in the same direction with both tools, but was statistically significant with only one of them; and Strong concordance if the results were fully aligned (significant/non-significant impact in same direction). For studies that measured HRQoL changes over time, it was determined whether the concordance between the generic and disease/condition-specific tool varied at different time points. Results were split into rare diseases non-rare diseases, and symptom-specific tools.

All analyses were performed using R 3.6.0 (Revolutions Analytics) and Microsoft Excel 365 (Microsoft).

\section{Results}

\section{Identification of disease- or condition-specific quality of} life tools

A total of 30,116 publications were reviewed from which 228 discreet, disease- or condition-specific HRQoL tools were identified (Fig. 1 \& Additional file 1). Full descriptions of 65 tools were unable to be sourced, either from published papers, online repositories, or via direct approaches to the authors. The remaining 163 tools (rare diseases: 17; non-rare: 75 ; symptom specific: 71 ) provided sufficient information/data for analysis, including 141 reporting results for a direct comparison against the SF-36 and/or EQ-5D (rare diseases: 10; non-rare: 73; symptom specific: 58 ). One tool (University of California Los Angeles-Prostate Cancer Index [UCLA-PCI]) completely overlaps with the SF-36, so was excluded from the SF-36 comparisons.

\section{Inclusion of appropriate domains and items SF-36}

The SF-36 had a sensitivity of $>75 \%$ against only one of the 162 disease/condition-specific HRQoL tools analysed, with a further five tools having a sensitivity between 50 and $75 \%$ (Additional file 2). A specificity of > $50 \%$ was achieved by the SF-36 against only one tool. The SF-36 did not achieve a sensitivity and specificity both $>50 \%$ against any of the $162 \mathrm{HRQoL}$ tools. For the 17 rare disease HRQoL tools, sensitivity ranged between 0 and $40 \%$ and specificity between 5 and $31 \%$. The corresponding rates for non-rare and symptom-specific tools were sensitivity: $0-100 \%$ and specificity: $0-100 \%$

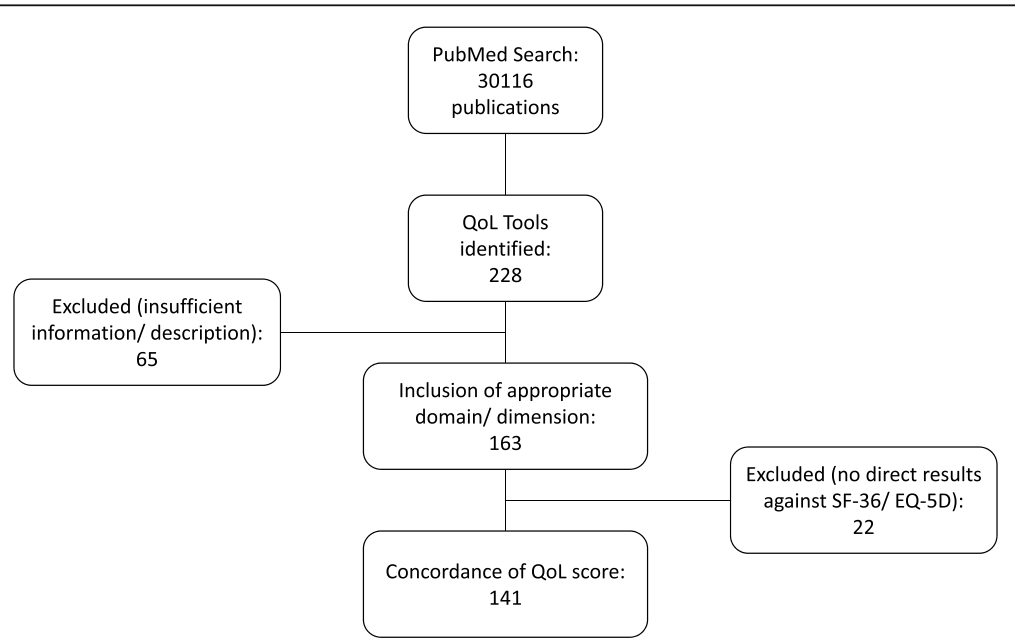

Fig. 1 Overview of identification and selection of disease- and condition-specific health-related quality of life tools 
and sensitivity: $0-60 \%$ and specificity: $0-19 \%$, respectively.

At a prevalence of poor $\mathrm{HRQoL}$ of $50 \%$, the proportion of false positives (FPR) was $>50 \%$ for $160 / 162$ of the disease/condition-specific HRQoL tools and $>75 \%$ for 137/162 tools against the SF-36 (Fig. 2). The corresponding false negative rate (FNR) was $>50 \%$ for $160 /$ 162 tools and $>75 \%$ for $159 / 162$ tools. Decreasing the prevalence of poor HRQoL to $20 \%$ increased the number of studies with a FPR of $>75 \%$ to $160 / 162$ tools, while the FNR was $<50 \%$ for $12 / 162$ tools. Conversely, increasing the prevalence of poor HRQoL to $80 \%$ resulted in $47 / 162$ tools having a FPR $<50 \%$, while $160 / 162$ tools had a FNR $>75 \%$.

\section{EQ-5D}

For the EQ-5D, a sensitivity of $>50 \%$ was not reached against any of the 163 disease/condition-specific HRQoL tools, with the highest recorded being $50 \%$ for one tool
(Additional file 2). A specificity of $>50 \%$ for the EQ-5D was found against $27 \mathrm{HRQoL}$ tools and $>75 \%$ against 13 tools. A sensitivity and specificity both $>50 \%$ was not achieved by the EQ-5D against any of the tools. Sensitivity and specificity ranged from 0 to $22 \%$ and $29-100 \%$, respectively, against rare tools, $0-50 \%$ and $0-100 \%$ for non-rare tools, and $0-25 \%$ and $0-100 \%$ for symptomspecific tools.

The FPR was $>50 \%$ for $156 / 163$ HRQoL tools and > $75 \%$ for $139 / 163$ HRQoL tools against the EQ-5D, when the prevalence of poor HRQoL was set at $50 \%$ (Fig. 3). Using the same prevalence of poor HRQoL, 156/163 tools had a FNR of $>50 \%$ and $49 / 163$ tools a FNR of $>$ $75 \%$. A prevalence of poor HRQoL of $20 \%$ increased the number of studies with a FPR of $>75 \%$ to $157 / 163$ tools, while the FNR was $<50 \%$ for $138 / 163$ tools. Increasing the prevalence of poor HRQoL to $80 \%$ resulted in $48 /$ 163 tools having a FPR $<50 \%$ and $162 / 163$ tools having a FNR $>75 \%$.
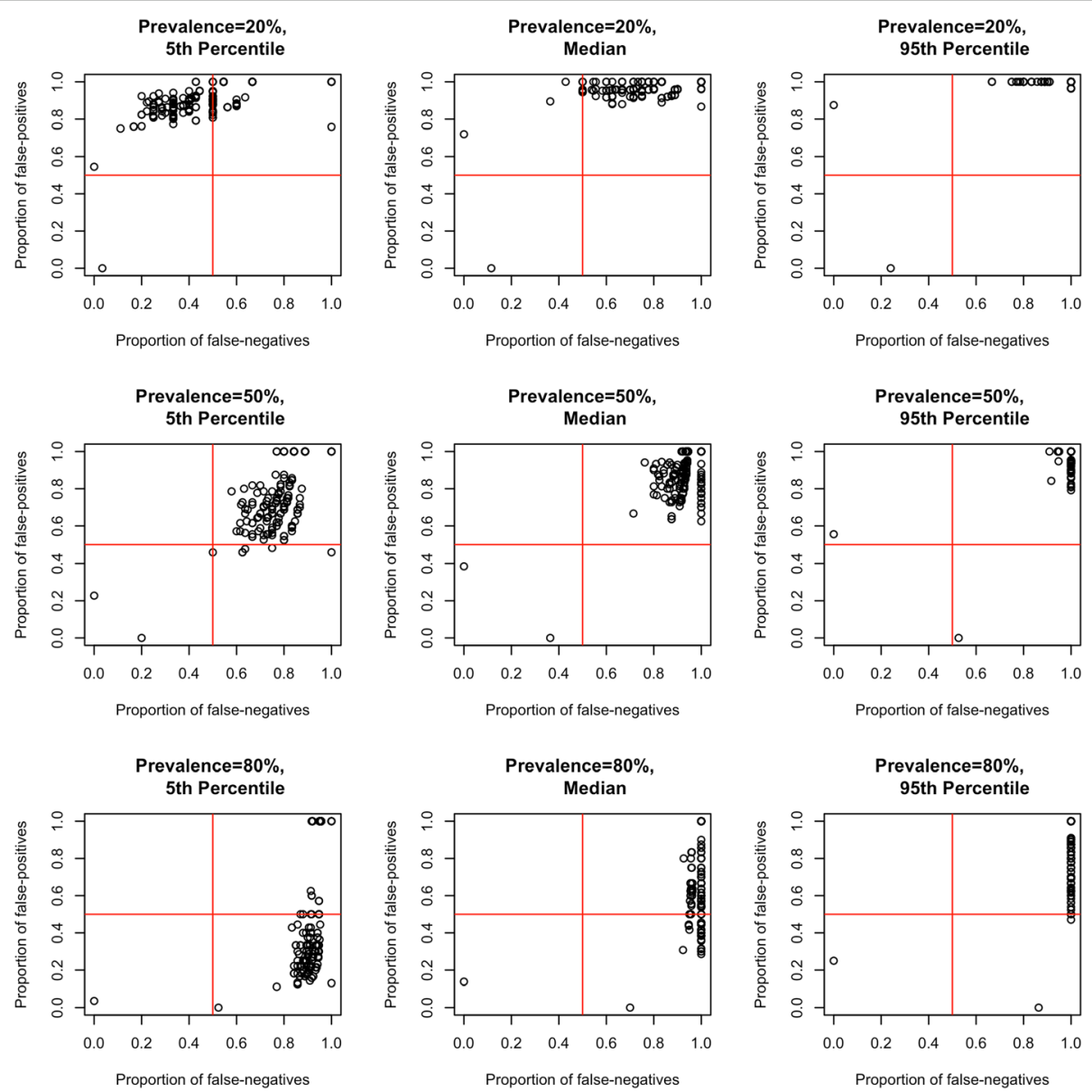

Fig. 2 Misclassification (false positives and false negatives) of all disease/condition-specific tools against the SF-36. The figure presents misclassification of all quality of life tools $(n=163)$ against the SF-36 by nine panels: three prevalence values (rows: 20,50, 80\%) and three points (columns: 5th percentile, median, 95th percentile of the prediction interval). Each point represents misclassification in 2 dimensions: proportions of false negatives (X-axis) and false positives ( $Y$-axis) 

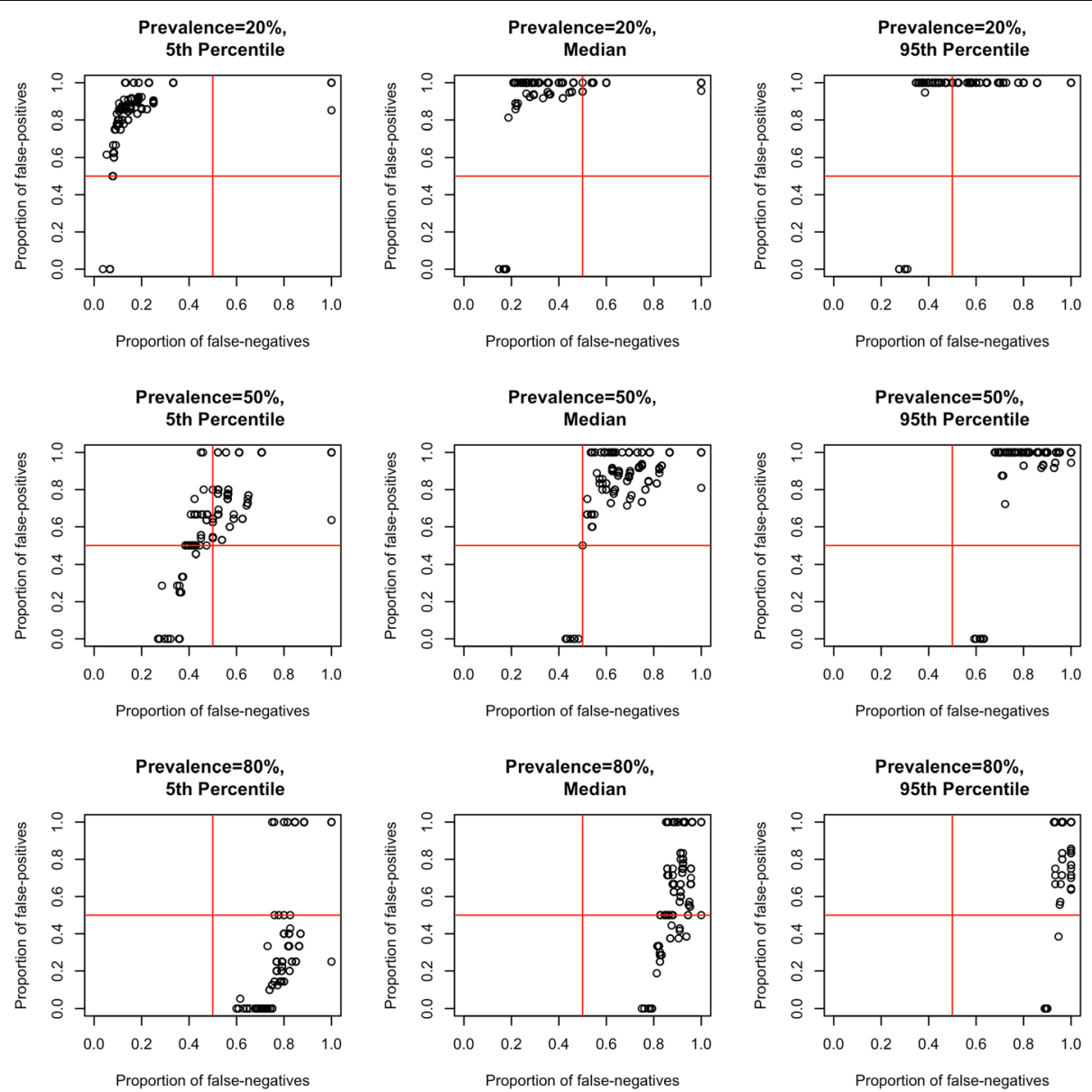

Fig. 3 Misclassification (false positives and false negatives) of all disease/condition-specific tools against the EQ-5D. The figure presents misclassification of all tools ( $n=163$ ) against EQ-5D by nine panels: three prevalence values (rows: 20,50, 80\%) and three points (columns: 5 th percentile, median, 95th percentile of the prediction interval). Each point represents misclassification in 2 dimensions: proportions of false negatives (X-axis) and false positives (Y-axis)

\section{Concordance of quality of life scores}

Of the disease/condition-specific HRQoL tools identified, 141 had comparative data directly against a generic tool (Fig. 1 \& Additional file 1). For rare diseases, 11 studies (including eight clinical trials) covering 10 tools provided data against the SF-36 (no comparative data against the EQ-5D was identified). One-hundred and twelve studies (including 74 clinical trials) covering 73 tools for non-rare diseases provided comparative data against the SF-36 (84 studies) and EQ-5D (65 studies), with some publications including data for both generic tools. Seventy-one studies (43 clinical trials) of 58 symptom-specific tools were available with data against the SF-36 (66 studies) and EQ-5D (31 studies), again with some studies covering both generic tools. Overall, $63 \%(125 / 198)$ of the publications reported results from clinical trials.

Strong concordance of the SF-36 results was reported for only $27 \%(3 / 11)$ of studies of rare disease tools, $29 \%$
(24/84) for non-rare disease tools, and 18\% (12/66) for symptom-specific tools (Table 1). Results were similar for the EQ-5D against non-rare tools, with 26\% (17/65) showing a Strong level of concordance, although this generic tool appeared to perform marginally better than the SF-36 against symptom-specific tools (29\%; 9/31). A total absence of concordance was noted for 30\% (25/84) and $35 \%(23 / 65)$ of results from studies of non-rare disease-specific tools and 36\% (24/66) and 16\% (5/31) for symptom-specific tools versus the SF-36 and EQ-5D, respectively.

For studies reporting HRQoL at multiple time points, a recorded change in HRQoL over time did not result in a reclassification of concordance for rare $(0 / 2)$ or nonrare disease tools $(0 / 34)$ versus the SF-36, but did for $19 \%(7 / 36)$ of symptom-specific tools (Table 2). For the EQ-5D, concordance was reclassified for $9 \%(2 / 23)$ of studies of non-rare disease tools, but not for symptomspecific tools $(0 / 7)$. 
Table 1 Concordance between health-related quality of life results for rare and non-rare disease and symptom-specific tools versus the SF-36 and EQ-5D

\begin{tabular}{|c|c|c|c|c|c|c|}
\hline \multirow{2}{*}{$\begin{array}{l}\text { Level of } \\
\text { Concordance }\end{array}$} & \multicolumn{2}{|l|}{ Rare Disease } & \multicolumn{2}{|c|}{ Non-rare Disease } & \multicolumn{2}{|c|}{ Symptom-specific } \\
\hline & SF-36 $(n=11)$ & EQ-5D $(n=0)$ & SF-36 $(n=84)$ & EQ-5D $(n=65)$ & SF-36 $(n=66)$ & EQ-5D $(n=31)$ \\
\hline None & $2(18 \%)$ & - & $25(30 \%)$ & $23(35 \%)$ & $24(36 \%)$ & $5(16 \%)$ \\
\hline Moderate & $6(55 \%)$ & - & $35(42 \%)$ & $25(38 \%)$ & $30(45 \%)$ & $17(55 \%)$ \\
\hline Strong & $3(27 \%)$ & - & $24(29 \%)$ & $17(26 \%)$ & $12(18 \%)$ & 9 (29\%) \\
\hline
\end{tabular}

Values represent number of studies (\% of total). Rare disease: affecting < 1 in 2000 population; Non-rare diseases: $\geq 1$ in 2000 population. Concordance: None $(\mathrm{No})=$ significant impact on quality of life with disease/condition specific tool, but no change or the opposite impact with generic tool (or vice versa); Moderate = quality of life impact was scored in the same direction with both tools, but statistically significant with only one of them; Strong= results fully aligned (significant/ non-significant impact in same direction)

\section{Discussion}

An accurate measure of HRQoL is of fundamental importance when considering the clinical- and costeffectiveness of a therapy or intervention during economic evaluations/HTAs to determine use within a healthcare system. Overestimating the impact on HRQoL could result in excessive healthcare expenditure for minimal health gain (money which could be better spent elsewhere). Conversely, underestimating the impact could cause unnecessary restrictions on use to the detriment of patients. Selection of the appropriate tool or tools to assess HRQoL is, therefore, essential. This study has found that by applying Neutral Theory, commonly used generic HRQoL tools, such as the SF-36 or EQ-5D, appear poorly aligned with disease- or condition-specific tools.

Neither the SF-36 nor the EQ-5D achieved a sensitivity and specificity for included items both $>50 \%$ against any of the 162/163 disease- or condition-specific tools included in this study. Even when using a high prevalence of poor HRQoL set at $80 \%$ (i.e. $4 / 5$ patients with this disease/condition have a notably impacted HRQoL), less than one-third of tools had a FPR of $<50 \%$ against the generic tools (SF-36: 29\% of tools; EQ-5D: 29\%). The situation was worse for rare disease tools, where sensitivity ranged from 0 to $40 \%$ for the SF-36 and $0-22 \%$ for the EQ-5D. Predicated on these results, it is unsurprising, therefore, that there were low levels of concordance between HRQoL scores from the generic versus the disease/condition-specific tools (no concordance vs SF-36: $18-36 \%$ of studies; vs EQ-5D: $16-35 \%$ ).

Salient limitations of this study included the necessity of having to assume a true negative fraction of 0.9 , as it was not possible to know if the disease/condition-specific tool fully captures all relevant items or domains (i.e., is completely Neutral). The measure of concordance between results of the generic and disease/conditionspecific tools was also necessarily crude to allow for cross comparison between multiple studies of numerous diseases/conditions. Importantly, however, a high number of studies (up to approximately one-third) reported zero concordance between generic and disease/condition tools. The use of the EQ-5D can also be considered a limitation in that this is not strictly a tool to measure HRQoL, but rather generic health status [6, 8]. The EQ$5 \mathrm{D}$ is, however, widely used to assess HRQoL $[9,10]$ and, therefore, was a valid choice for this study. It is also worthy of note that, surprisingly, a full description of $29 \%(65 / 228)$ of the identified tools could not be obtained, despite their publication in indexed journals. This is an unacceptably high rate; such descriptions should be a standard component of publication.

HRQoL tools generate scores on the basis of individual item measures - a construct. The concept of 'True' HRQoL at any given time is, therefore, important. The tools generate a value of observed HRQoL on a subject based on relevant items and lack of irrelevant items. The principle underpinning the development and use of disease- and condition-specific tools is that they are inherently more accurate than generic tools at measuring HRQoL for patients with that particular disease or condition. Thereby, closer to neutrality. However, tools have been developed for the same disease/condition that do not include all the same items and domains [13, 14]. This raises the question of what is the correct construct to ensure an accurate assessment of HRQoL for that

Table 2 Change in health-related quality of life over time and impact on concordance of results between rare and non-rare and symptom-specific tools versus the SF-36 and EQ-5D

\begin{tabular}{|c|c|c|c|c|c|c|}
\hline & \multicolumn{2}{|l|}{ Rare Disease } & \multicolumn{2}{|c|}{ Non-rare Disease } & \multicolumn{2}{|c|}{ Symptom-specific } \\
\hline & SF-36 $(n=2)$ & EQ-D $(n=0)$ & SF-36 $(n=38)$ & EQ-5D $(n=27)$ & SF-36 $(n=42)$ & EQ-5D $(n=7)$ \\
\hline QoL change & $2(100 \%)$ & - & $34(89 \%)$ & $23(85 \%)$ & $36(86 \%)$ & 7 (100\%) \\
\hline Concordance change & $0 / 2(0 \%)$ & - & 0/34 (0\%) & 2/23 (9\%) & 7/36 (19\%) & $0 / 7(0 \%)$ \\
\hline
\end{tabular}

$n=$ studies with $>1$ time point. Rare disease: affecting $<1$ in 2000 population; Non-rare diseases: $\geq 1$ in 2000 population 
disease/condition. Several approaches have been taken to improve the accurate assessment of HRQoL, including: the parallel use of generic and disease/condition tools $[15,16]$; using mapping algorithms from disease/ condition-specific tools to generic tools [10]; tailoring standard items to specific diseases/conditions [17]; and use of bolt-on items to generic questionnaires [10]. Despite these approaches, the pertinent question remains what is acceptable accuracy for a HRQoL tool? Given the importance of having an accurate measure of the impact of a therapy or intervention on HRQoL, may be it is time for there to be rethink on how HRQoL is assessed and measured. Moreover, to consider how improvements can be made to the current widespread use of generic tools.

\section{Conclusions}

A new theory recently proposed is that of a 'Neutral Observer', which provides a set of principles on which a determination of the accuracy of an observation of a given construct can be based [1]. It is theorised that the "true" value of a construct can be measured by an abstract or Neutral observer who has access to a complete list of indicators that are all relevant to the empirical measurement of a construct. This Neutral Observation thereby serves as the reference against which observations using the construct can be assessed for accuracy [1]. Adoption of such an approach in the development and assessment of HRQoL tools could improve their relevance, accuracy, and utility in economic evaluations of health interventions.

\section{Abbreviations}

Cl: Confidence interval; EQ-5D: EuroQol five-dimensional; FNR: False negative rate; FPR: False positive rate; HRQoL: Health-related quality of life; HTA: Health technology appraisal; NICE: National Institute for Health and Care Excellence; SF-36: Medical Outcomes Study Short Form family of measures; UCLAPCl: University of California Los Angeles-Prostate Cancer Index; WHO: World Health Organization

\section{Supplementary Information}

The online version contains supplementary material available at https://doi. org/10.1186/s12874-021-01279-w.

Additional file 1. Details of all included and excluded tools. Additional file 2. Results for inclusion of appropriate domains and items for all assessed tools vs SF-36 and EQ-5D.

\section{Acknowledgements}

Jesica MacLeod (Medalis) provided support for the literature searches and evidence review, sourcing the tools, and data extraction. Solomon Christopher (Medialis) conducted all the statistical analyses.

\section{Author's contributions}

RJ led and came up with the concept of the study and authored and approved the manuscript.

\section{Authors' information}

$\mathrm{RJ}$ is a pharmaceutical physician specialising in medical affairs and is a visiting senior lecturer at the Pharmaceutical Medicine Research Centre, King's College London. RJ is the author of the Jandhyala method in observing consensus of answers to list-generating questioning and has researched other methods of generating real-world evidence through development of disease specific measurement tools and core datasets. He has a special interest in the observation of the disease phenotype of subjects recruited to clinical studies.

\section{Funding}

No external funding sources supported this work.

\section{Availability of data and materials}

The datasets used and/or analysed during the current study are available from the corresponding author on reasonable request.

\section{Declarations}

Ethics approval and consent to participate Not applicable.

\section{Consent for publication}

Not applicable.

\section{Competing interests}

The author declares that he has no competing interests.

Received: 19 December 2020 Accepted: 8 April 2021

Published online: 26 April 2021

\section{References}

1. Neutral theory: a conceptual framework for construct measurement in clinical research. https://doi.org/10.13140/RG.2.2.33212.16008. (Pre-print)

2. World Health Organization (WHO). WHOQOL: Measuring Quality of Life. Available at: https://www.who.int/healthinfo/survey/whoqol-qualityoflife/ en/. Accessed 18 Dec 2020.

3. Hays RD, Reeve BB. Measurement and modeling of health-related quality of life. In: Heggenhougen HK, Quah S, editors. International encyclopedia of public health. London: Academic Press; 2008. p. 241-52. https://doi.org/10.1 016/B978-012373960-5.00336-1.

4. Ware JE Jr, Sherbourne CD. The MOS 36-item short-form health survey (SF36). I. Conceptual framework and item selection. Med Care. 1992;30(6):47383. https://doi.org/10.1097/00005650-199206000-00002.

5. RAND Corporation. 36-Item Short Form Survey (SF-36). https://www.rand. org/health-care/surveys_tools/mos/36-item-short-form.html. Accessed 14 Mar 2021.

6. Rabin R, de Charro F. EQ-5D: a measure of health status from the EuroQol group. Ann Med. 2001;33(5):337-43. https://doi.org/10.3109/078538901 09002087.

7. EuroQol. Eq-5D. https://euroqol.org/. Accessed 14 Mar 2021

8. Karimi M, Brazier J. Health, health-related quality of life, and quality of life: what is the difference? Pharmacoeconomics. 2016;34(7):645-9. https://doi. org/10.1007/s40273-016-0389-9.

9. National Institute for Health and Care Excellence (NICE). Guide to the methods of technology appraisal 2013. https://www.nice.org.uk/process/ pmg9/chapter/foreword. Accessed 14 Mar 2021.

10. Longworth L, Yang Y, Young T, Mulhern B, Hernández Alava M, Mukuria C, et al. Use of generic and condition-specific measures of health-related quality of life in NICE decision-making: a systematic review, statistical modelling and survey. Health Technol Assess. 2014;18(9):1-224. https://doi. org/10.3310/hta18090

11. Ouzzani M, Hammady H, Fedorowicz Z, Elmagarmid A. Rayyan-a web and mobile app for systematic reviews. Syst Rev. 2016;5(1):210. https://doi.org/1 0.1186/s13643-016-0384-4

12. Baldovino S, Moliner AM, Taruscio D, Daina E, Roccatello D. Rare diseases in Europe: from a wide to a local perspective. Isr Med Assoc J. 2016;18(6):359-63.

13. Chen X-L, Zhong L-H, Wen Y, Liu TW, Li XY, Hou ZK, et al. Inflammatory bowel disease-specific health-related quality of life instruments: a systematic review of measurement properties. Health Qual Life Outcomes. 2017;15(1): 177. https://doi.org/10.1186/s12955-017-0753-2. 
14. Marinus J, Ramaker C, van Hilten JJ, Stiggelbout AM. Health related quality of life in Parkinson's disease: a systematic review of disease specific instruments. J Neurol Neurosurg Psychiatry. 2002;72(2):241-8. https://doi. org/10.1136/jnnp.72.2.241.

15. Assari S, Lankarani MM, Montazeri A, Soroush MR, Mousavi B. Are generic and disease-specific health related quality of life correlated? The case of chronic lung disease due to sulfur mustard. J Res Med Sci. 2009;14(5):285-90.

16. Seow LSE, Tan THG, Abdin E, Chong SA, Subramaniam M. Comparing disease-specific and generic quality of life measures in patients with schizophrenia. Psychiatry Res. 2019;273:387-93. https://doi.org/10.1016/j. psychres.2019.01.034

17. Ware JE Jr, Gandek B, Guyer R, Deng N. Standardizing disease-specific quality of life measures across multiple chronic conditions: development and initial evaluation of the QOL disease impact scale (QDIS ${ }^{\oplus}$ ). Health Qual Life Outcomes. 2016;14(1):84. https://doi.org/10.1186/s12955-016-0483-x.

\section{Publisher's Note}

Springer Nature remains neutral with regard to jurisdictional claims in published maps and institutional affiliations.

Ready to submit your research? Choose BMC and benefit from:

- fast, convenient online submission

- thorough peer review by experienced researchers in your field

- rapid publication on acceptance

- support for research data, including large and complex data types

- gold Open Access which fosters wider collaboration and increased citations

- maximum visibility for your research: over $100 \mathrm{M}$ website views per year

At BMC, research is always in progress.

Learn more biomedcentral.com/submissions 